\title{
Modeling of Processes Taking Place during Powder Coating Treatment by an Electron Beam or a Plasma Jet
}

\author{
D. Alontseva ${ }^{a, *}$, A. Krasavin ${ }^{a}$, T. Kolesnikova ${ }^{a}$ And A. Russakova ${ }^{b}$ \\ ${ }^{a}$ East-Kazakhstan State Technical University, Protazanov 69, 070004, Ust-Kamenogorsk, Kazakhstan \\ ${ }^{b}$ Eurasian National University, Munaitpasov 5, 01008, Astana, Kazakhstan
}

\begin{abstract}
This paper considers the problem of finding the temperature field in two-layer metallic materials heated by a moving source of radiation. It describes developed by the authors numerical method for solving the problem of heating a two-layer plate by a moving axially symmetric surface heat source with regard to the function of distribution of the power density of the beam for which the program of computation in $\mathrm{C}^{++}$was implemented. The calculation results were used for selecting the optimal parameters (speed and power density of the source) of modifying radiation of protective powder coatings on steel substrates.
\end{abstract}

DOI: 10.12693/APhysPolA.125.1275

PACS: $02.60 . \mathrm{Cb}, 02.30 . \mathrm{Nw}, 44.05 .+\mathrm{e}$

\section{Introduction}

The relevance of the problem of finding the temperature field in two-layer metal materials heated by a moving source of radiation is conditioned by the problem of choosing the optimal modes of modifying radiation of protective powder coatings applied by a high-speed plasma jet to the steel substrate. Based on a series of experimental studies of the structure and properties of such coatings before and after additional irradiation by electron beam or plasma jet [1-5], we concluded that it is necessary to simulate the temperature distribution during irradiation to justify the choice of such irradiation parameters as power density and the speed of the source. The values and the distribution of temperature in the depth from the surface heated by a source play a crucial role in the precipitation of reinforcing phase particles from the solid solution, acceleration of diffusion processes between the coating and the substrate. Comparing the experimental data with the temperature profile calculations we can offer the best modes of radiation that do not cause excessive heating of a coating, but at the same time lead to changes in the phase composition (formation of reinforcing particles) and to improvement of adhesion of coatings to the substrate due to acceleration of diffusion processes between the coating and the substrate.

During radiation treatment of coatings high temperatures of the coating surface are achieved (the melting of the coating surface is an often phenomenon), at that the temperature of the substrate at a relatively small depth increases slightly. Since the thermal properties of metals, such as thermal conductivity and specific thermal capacitance, substantially depend on temperature, adequate modeling of heat transfer in the processing of coatings

*corresponding author; e-mail: dalontseva@mail.ru by radiation leads to the solution of the non-stationary problem of the theory of non-linear thermal conductivity. Problems of the nonlinear theory of heat conductivity are solved almost exclusively by numerical methods due to the extreme difficulty of finding analytical solutions of emerging problems $[6,7]$. From a practical point of view, it is essential to consider the following fact: a mathematically rigorous proof of correctness of approximate methods used for solving nonlinear heat transfer problems in most cases is not available; and so far the main criterion for the suitability of one or the other approximate method is its try-out in model problems solved by classical methods of linear theory of heat conduction. It is clear that trying-out in model problems is also needed in the development of software designed for the simulation of heat transfer processes. Thus, the solution of problem of linear thermal conductivity of heating a two-layer plate by moving surface heat source is necessary to address a much more challenging task of modeling of heat transfer processes at the radiation treatment of coatings. In addition, the solution of this problem is useful for selecting the "first targets" in selecting the operational parameters of irradiation (power and speed of the beam), and therefore is of separate interest.

A number of contemporary works that address thematically similar problems [8-14], on the one hand, show the relevance of the problem of finding the distribution of temperature during irradiation by beams of charged particles; on the other hand, show a different approach to solving the problem of heat conductivity, a nonlinear heat conduction problem is solved by the finite element method (FEM). Since FEM is very sensitive to the choice of area division, and the theoretical error estimate is very difficult, in practice successful application of FEM often depends on the experience and intuition of a researcher, and the main criterion for the plausibility of the results is still an experiment. Experimental confirmation of numerical calculations involves great technical difficulties, so the solution of the given model problem by classical 
methods of linear thermal conductivity, in our view, is a necessary step in the development of a complete model of behavior of material heated by a source of radiation.

In this paper we propose the developed numerical method for solving the problem of heating a two-layer plate by a moving axially symmetric surface heat source. The following is the description of the proposed method.

\section{Physical and mathematical formulation of the problem}

Since the thickness of the coating layer in which heat is emitted during radiation processing is very small compared to the thickness of the coating, we use a flat heat source model. Because in most practical cases geometric dimensions of the zone of intense heating are small compared to the size of a sample, we simulate the substrate by a semi-infinite body, and the coating - by an unlimited plate of thickness $h$. We introduce the Cartesian coordinates as shown in Fig. 1 ( $X$ and $Y$ axes are lying in the plane of the coating surface, $Z$ axis is pointed into the sample). Let at the initial time $t_{0}$ a flat axially symmetric heat source moving with velocity $V$ directed along the axis $X$ be switched; and at the time $t_{0}$ the axis of the beam passes through the point $\left(x_{0}, 0,0\right)$ where $x_{0}=V t_{0}$.

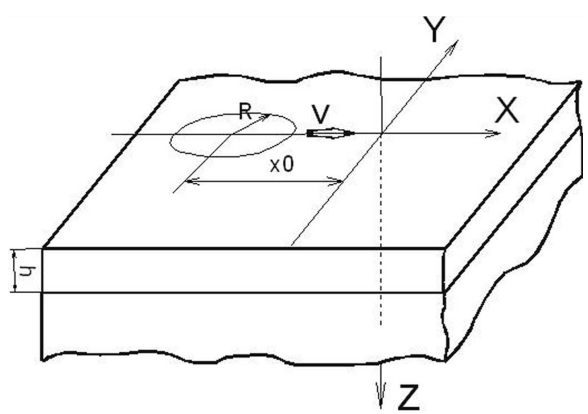

Fig. 1. Schematic representation of the bilayer plate heated by the moving flat heat source, indicating the choice of coordinate system.

Then we come to the boundary problem of the theory of thermal conductivity defined by the system of differential equations and boundary conditions:

$$
\left\{\begin{array}{l}
\frac{\partial^{2} T_{i}}{\partial x^{2}}+\frac{\partial^{2} T_{i}}{\partial y^{2}}+\frac{\partial^{2} T_{i}}{\partial z^{2}}=\frac{1}{a_{i}} \frac{\partial T_{i}}{\partial t} \\
T_{i}\left(x, y, z, t_{0}\right)=0 \\
\left(\frac{\partial T_{1}}{\partial z}\right)_{z=0}=f(x, y, t) \\
k_{1}\left(\frac{\partial T_{1}}{\partial z}\right)_{z=h}=k_{2}\left(\frac{\partial T_{2}}{\partial z}\right)_{z=h}, \\
T_{1}(x, y, h, t)=T_{2}(x, y, h, t)
\end{array}\right.
$$

where $T_{1}(x, y, z, t)$ and $T_{2}(x, y, z, t)$ are thermal fields in the coating and the substrate; $a_{1}$ and $k_{1}$ - coefficients of thermal diffusivity and conductivity of the coating material, $a_{2}$ and $k_{2}$ - coefficients of thermal diffusivity and thermal conductivity of the substrate material. The surface heat source is described by the equation

$$
f(x, y, t)=Q(r(x, y, t)) \varphi\left(t-t_{0}\right),
$$

in which, $r(x, y, t)=\sqrt{\left[x-x_{0}-V\left(t-t_{0}\right)\right]^{2}+y^{2}}, Q(r)$
- the function that describes the distribution of the source power surface density (argument $r$ sets the distance to the axis of the source), and $\phi(t)$ function is given by the system

$$
\varphi(t)=\left\{\begin{array}{l}
0, t<0, \\
1, t \geq 0 .
\end{array}\right.
$$

\section{Solution method}

Let a fixed flat heat source with a power density (4) operate on the surface of the above-described composite solid

$$
N(r, t)=Q(r) \varphi\left(t-t_{0}\right) .
$$

If we can calculate the temperature field $T^{*}(r, z, t)$ (in a cylindrical coordinate system, $z$ axis of which coincides with the axis of the source and is pointed into the plate) generated by the source, we will be able to find the field of a moving source by calculating the quadratures. Indeed, the temperature at the sample heated moving source at the time is determined by Eq. (5) [7]. In point of fact, the temperature $T_{A}$ at the point $A(x, y, z)$ of the sample heated by a moving source at the time $t$ is determined by Eq. (5) [7]:

$$
T_{A}=\int_{t_{0}}^{t}\left(\frac{\partial T^{*}\left(\sqrt{\left[x-x_{0}-u\left(\tau-t_{0}\right)\right]^{2}+y^{2}}, z, \tau\right)}{\partial \tau}\right) \mathrm{d} \tau .
$$

To find the field $T^{*}(r, z, t)$ we consider the following problem of thermal conductivity: there is a composite solid, which is a semi-infinite cylinder of radius $R$ (a region of space defined by the inequalities $r \leq R, z>h$ ), whose material has a thermal conductivity coefficient $k_{2}$ and the coefficient of thermal diffusivity $a_{2}$, in contact with the cylinder of radius $R$ and height $h$, made of material with thermal conductivity and thermal diffusivity $k_{1}$ and $a_{1}$, respectively. At the initial time an axially symmetric flat heat source with a surface power density given by the function $N(r, t)=Q(r) \varphi(t)$ starts to act on the surface of the body; the temperature of the side surface of the composite cylinder is maintained at zero. The body temperature at the initial time is zero. Find the temperature field of the upper cylinder defined by the function $T_{1}(r, z, t)$ and the temperature field in a semi-infinite cylinder - function $T_{2}(r, z, t)$.

We may assume that if the function $Q(r)$ is finite, i.e. there is such $r_{\max }$ that $Q(r)=0$ if $r>r_{\max }$, and $r_{\max } \ll R$, then the solution will be a good approximation to the field $T^{*}\left(r, z, t-t_{0}\right)$. We approximate the function $Q(r)$ by the first $N$ members of its expansion in a Fourier-Bessel with Bessel functions $J_{0}(r)$ :

$$
Q(r) \approx \sum_{k=0}^{N} a_{k} J_{0}\left(\zeta_{k} r\right),
$$

where $\zeta_{k}=\frac{\lambda_{k}}{R}\left(\lambda_{k}-k\right.$-th root of the function $J_{0}(r)$ ). The solution of the problem takes the form $T_{1}=\sum_{k=0}^{N} a_{k} T_{1 k}, T_{2}=\sum_{k=0}^{N} a_{k} T_{2 k}$, where functions $T_{1 k}$ and $T_{2 k}$ satisfy the differential equation of heat conductivity $\Delta T_{i k}=\frac{1}{a_{i}} \frac{\partial T_{i k}}{\partial t}$, boundary conditions $k_{1}\left(\frac{\partial T_{1 k}}{\partial z}\right)_{z=0}=-\varphi(t) J_{0}\left(\zeta_{k} r\right), k_{1}\left(\frac{\partial T_{1 k}}{\partial z}\right)_{z=h}=$ 
$k_{2}\left(\frac{\partial T_{2 k}}{\partial z}\right)_{z=h}, \quad T_{1 k}(r, h, t)=T_{2 k}(r, h, t)$, as well as the condition $T_{2 k}(r, \infty, t)=0$ and initial conditions $T_{1 k}(r, z, 0)=0$ and $T_{2 k}(r, z, 0)=0$. We perform Laplace transformation with respect to the variable $t$, having designated $\bar{T}_{1 k}(s, r, z)=\int_{0}^{\infty} T_{1 k}(r, z, t) \mathrm{e}^{-s t} \mathrm{~d} t$ and $\bar{T}_{2 k}(s, r, z)=\int_{0}^{\infty} T_{2 k}(r, z, t) \mathrm{e}^{-s t} \mathrm{~d} t$.

In the future we will denote the correspondence of the original to the image at the Laplace transformation by the icon $\rightarrow$, for example, $T_{1 k} \rightarrow \bar{T}_{1 k}$. The differential equations of heat conductivity for the originals pass into the equations of the eigenfunctions of the Laplace operator $\Delta \bar{T}_{1 k}=\frac{s}{a_{1}} \bar{T}_{1 k}$ and $\Delta \bar{T}_{2 k}=\frac{s}{a_{2}} \bar{T}_{2 k}$. Note that the functions $f(s, r, z)=J_{0}\left(\zeta_{k} r\right) \exp (\eta z)$ and $g(s, r, z)=J_{0}\left(\zeta_{k} r\right) \exp (-\eta z)$, where $\eta=\sqrt{\zeta_{k}^{2}+\frac{s}{a}}$ will be the eigenfunctions of the Laplace operator with eigenvalue $\frac{s}{a}$. We look for $\bar{T}_{1 k}$ and $\bar{T}_{2 k}$ as

$$
\begin{aligned}
& \bar{T}_{1 k}=J_{0}\left(\zeta_{k} r\right)\left[f_{1 k} \exp \left(\eta_{1 k} z\right)+f_{2 k} \exp \left(-\eta_{1 k} z\right)\right], \\
& \bar{T}_{2 k}=J_{0}\left(\zeta_{k} r\right) f_{3 k} \exp \left(-\eta_{2 k}(z-h)\right),
\end{aligned}
$$

where $\eta_{1}=\sqrt{\zeta_{k}^{2}+\frac{s}{a_{1}}}$ [15]. Taking into account that $\varphi(t) \rightarrow \frac{1}{s}$ [16], the boundary conditions give a system of equations for determining $f_{1}, f_{2}, f_{3}$ :

$$
\left\{\begin{array}{l}
k_{1} \eta_{1 k} f_{1 k}-k_{1} \eta_{1 k} f_{2 k}=-\frac{1}{s} \\
\exp \left(h \eta_{1 k}\right) f_{1 k}+\exp \left(-h \eta_{1 k}\right) f_{2 k}=f_{3 k} \\
k_{1} \eta_{1 k} \exp \left(h \eta_{1 k}\right) f_{1 k}-k_{1} \eta_{1 k} \exp \left(-h \eta_{1 k}\right) f_{2 k} \\
\quad=-k_{2} \eta_{2 k} f_{3 k},
\end{array}\right.
$$

solving which we get

$$
\begin{aligned}
f_{3 k} & =\frac{1}{s\left(k_{1} \eta_{1 k} \operatorname{sh}\left(\eta_{1 k} h\right)+k_{2} \eta_{2 k} \operatorname{ch}\left(\eta_{1 k} h\right)\right)}, \\
f_{1 k} & =\frac{k_{1} \eta_{1 k}-k_{2} \eta_{2 k}}{2 k_{1} \eta_{1 k}} \exp \left(-h \eta_{1 k}\right) f_{3 k}, \\
f_{2 k} & =\frac{k_{1} \eta_{1 k}+k_{2} \eta_{2 k}}{2 k_{1} \eta_{1 k}} \exp \left(h \eta_{1 k}\right) f_{3 k} .
\end{aligned}
$$

As for the estimation of the temperature field of a moving source, we need not the temperature fields $T_{1}(r, z, t)$ and $T_{2}(r, z, t)$ as such, but their time derivatives $\frac{\partial T_{1}(r, z, t)}{\partial t}$ and $\frac{\partial T_{2}(r, z, t)}{\partial t}$, we use the property of the Laplace transformation: if $f(t) \rightarrow F(s)$, then $\frac{\mathrm{d} f(t)}{\mathrm{d} t} \rightarrow s F(s)-f(0)$. From the initial conditions (11) and (12) we get the view

$$
\begin{aligned}
& \frac{\partial T_{1}(r, z, t)}{\partial t}=\sum_{k=0}^{N} a_{k} J_{0}\left(\zeta_{k} r\right) y_{1 k}(t), \\
& \frac{\partial T_{2}(r, z, t)}{\partial t}=\sum_{k=0}^{N} a_{k} J_{0}\left(\zeta_{k} r\right) y_{2 k}(t),
\end{aligned}
$$

where

$$
\begin{aligned}
& y_{1 k}(t) \rightarrow F_{1 k} \exp \left(\eta_{1 k} z\right)+F_{2 k} \exp \left(-\eta_{1 k} z\right), \\
& y_{2 k}(t) \rightarrow Y_{k} \exp \left(-\eta_{2 k}(z-h)\right),
\end{aligned}
$$

and $Y_{k}, F_{1 k}$, and $F_{2 k}$ are defined by the equations:

$$
Y_{k}=\frac{1}{k_{1} \eta_{1 k} \operatorname{sh}\left(\eta_{1 k} h\right)+k_{2} \eta_{2 k} \operatorname{ch}\left(\eta_{1 k} h\right)},
$$

$$
\begin{aligned}
& F_{1 k}=\frac{k_{1} \eta_{1 k}-k_{2} \eta_{2 k}}{2 k_{1} \eta_{1 k}} \exp \left(-h \eta_{1 k}\right) Y_{k}, \\
& F_{2 k}=\frac{k_{1} \eta_{1 k}+k_{2} \eta_{2 k}}{2 k_{1} \eta_{1 k}} \exp \left(h \eta_{1 k}\right) Y_{k} .
\end{aligned}
$$

As it is known, when solving heat conductivity problems with the use of the Laplace transformation in time the greatest challenge is to transform the inverse transformation [15-17]. In this case, a key role is played by the problem of finding the original of the function (17). For simplicity we shall omit the index $k$, and will take the image $Y(s)=\frac{1}{k_{1} \eta_{1} \operatorname{sh}\left(\eta_{1} h\right)+k_{2} \eta_{2} \operatorname{ch}\left(\eta_{1} h\right)}$.

In fact, knowing the original of the image $y(t)$ it is easy to find $y_{2}(t)$ as the convolution of functions $y(t)$ of the original image.

$$
G(s)=\exp \left(-\eta_{2}(z-h)\right), \quad g(z, t) \rightarrow \exp \left(-\eta_{2}(z-h)\right) .
$$

To find the original of $Y(s)$ the following method was used: we will write $Y(s)$ in the form

$$
Y(s)=\frac{1}{\alpha \theta_{1} \operatorname{sh}\left(\gamma \theta_{1}\right)+\beta \theta_{2} \operatorname{ch}\left(\gamma \theta_{1}\right)},
$$

where $\alpha=\frac{k_{1}}{\sqrt{a_{1}}}, \beta=\frac{k_{2}}{\sqrt{a_{2}}}, \gamma=\frac{h}{\sqrt{a_{1}}}, \theta_{1}=\sqrt{\zeta_{k}^{2} a_{1}+s}$ and $\theta_{2}=\sqrt{\zeta_{k}^{2} a_{2}+s}$. We introduce the function

$$
Y^{*}[s]=\frac{1}{\alpha \theta_{1} \operatorname{sh}\left(\gamma \theta_{1}\right)+\beta \theta_{1} \operatorname{ch}\left(\gamma \theta_{1}\right)}
$$

and represent $Y(s)$ in the form

$$
Y(s)=Y^{*}(s)+D(s) \text {. }
$$

It can be proved that the function $D(i t)$ of a real argument $t$ is square integrable, and because of this, it can be regarded as the Fourier spectrum of some function $d(t)$. In such a case, the original image $Y(s)$ can be found as

$$
y(t)=y^{*}(t)+d(t),
$$
where $y^{*}(t)$ is the original image $Y^{*}(s)$. It should be noted that at close to each other values of coating and substrate materials thermal conductivity coefficients the function $Y^{*}(s)$ accurately approximates the function $Y(s)$ in the right half-plane; and we can assume that $y(t) \approx y^{*}(t)$.

The original image $Y^{*}(s)$ can be found analytically. To do this, we represent $Y^{*}(s)$ in the form

$$
Y^{*}(s)=\frac{1}{\nu \theta_{1} \exp \left(\gamma \theta_{1}\right)\left(1+\varepsilon \exp \left(-2 \gamma \theta_{1}\right)\right)},
$$

where $\varepsilon=\frac{\mu}{\nu}$, and put the expression $\frac{1}{1+\varepsilon \exp \left(-2 \gamma \theta_{1}\right)}$ as a sum of geometric progression. Then

$$
\begin{aligned}
Y^{*}(s) & =\frac{1}{\nu}\left(\frac{\exp \left(-\gamma \theta_{1}\right)}{\theta_{1}}\right. \\
+\sum_{k=1}^{n} & \left.\frac{(-\varepsilon)^{k} \exp \left(-(2 k+1) \gamma \theta_{1}\right)}{\theta_{1}}\right),
\end{aligned}
$$

from which we obtain

$$
y(t)=\frac{1}{\nu} \sum_{k=0}^{n} \frac{(-\varepsilon)^{k} \exp \left(-\frac{(2 k+1) \gamma}{4 t}-(2 k+1) \zeta_{k}^{2} a_{1} t\right)}{\sqrt{4 \pi t}} .
$$

Thus, the original $Y(s)$ is found as the sum of the form (24), where the function $d(t)$ is calculated by numer- 
ical inversion of the Fourier transform of function $D(i t)$.

\section{Implementation and practical application of the method}

The method is implemented in the program for the $\mathrm{PC}$ written in the $\mathrm{C}^{++}$programming language in Microsoft Visual Studio Express development environment.

Figure 2 provides screenshots taken during the operation of the program implementing the above method of calculation of the thermal field of a moving source with the following inputs: heat source - Gaussian distribution of surface power density; effective beam diameter $10 \mathrm{~mm}$; power source - $500 \mathrm{~W}$; speed $-4 \mathrm{~mm} / \mathrm{s}$; coating - $300 \mu \mathrm{m}$ thick; material — nickel; density $8900 \mathrm{~kg} / \mathrm{m}^{3}$, the thermal conductivity of $90.4 \mathrm{~W} / \mathrm{mK}$; specific heat capacity $443 \mathrm{~J} / \mathrm{kg}$. Substrate: material — iron; density $7870 \mathrm{~kg} / \mathrm{m}^{3}$; thermal conductivity $79.9 \mathrm{~W} / \mathrm{mK}$; specific heat capacity $447.0 \mathrm{~J} /(\mathrm{kg} \mathrm{K})$.

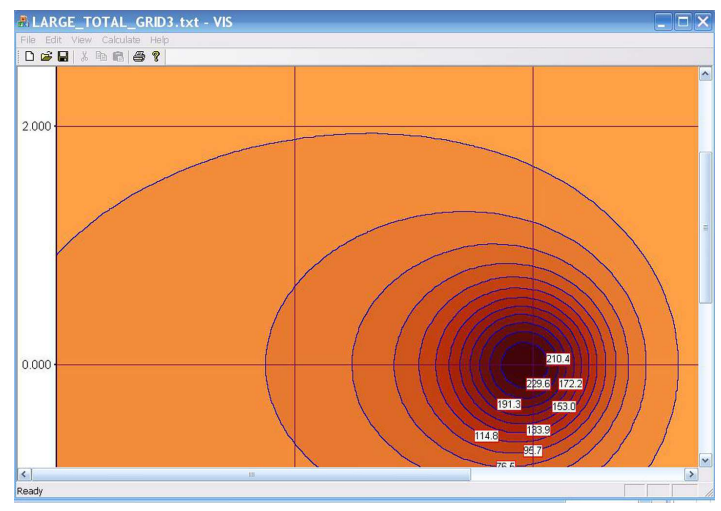

Fig. 2. Contour maps of temperature field on the border of the coating and the substrate (horizontal section).

Based on calculations of temperature profiles for bilayer absorbers wherein the coating layer has been interpreted as nickel or cobalt, and the substrate - as iron, we have proposed certain modes of modification of protective powder $\mathrm{Ni}$ and Co-based coatings deposited by a high-speed plasma jet onto the steel substrates. Justification of interpretation of multi-component coatings on a steel substrate by $\mathrm{Ni}-\mathrm{Fe}$ and $\mathrm{Co}-\mathrm{Fe}$ bilayer absorbers is based on the data of experimental studies of structural and phase composition of these coatings and the developed model of their structure $[1,3,4]$. We selected the modes of further processing by a plasma jet leading to melting of the surface layer of the coating without melting the coating to its full depth. Improved wear resistance of the modified coatings was achieved by reducing the surface roughness at melting, and improved adhesion of the coating to the substrate - by accelerating diffusion processes during irradiation $[1,5]$. The experimental results of the study of the structure and properties of modified by the estimated modes surfaces are confirmed by the two acts on production testing at the Sumy Institute for Surface Modification (Sumy, Ukraine) and are in good agreement with the data of other researchers. In particular, the papers $[10,18]$ experimentally proved the formation of hardening intermetallic compounds during coating irradiation; and the monograph [19] confirmed the development of radiation-induced diffusion in materials with the fcc lattice during irradiation, which leads to heating up to temperatures we calculated.

\section{Conclusions}

Presented in this paper solution of the problem of linear thermal conductivity on a two-layer plate heated by a moving surface heat source, firstly, was used to select the process parameters (power density and velocity of the beam) for the process of modifying irradiation of protective powder coatings by the electron beam and by a plasma jet. As shown in our papers $[1,2,5]$, in the result of treatment according to the recommended modes the increased product durability was achieved by melting coating layer surface (reduction in roughness of coatings), improved coating adhesion to the substrate (acceleration of diffusion processes between the coating and the substrate), and an increase in microhardness of the transition layer from the substrate to the coating by precipitation of strengthening intermetallic phases. The results confirm the correctness of calculations of temperature profiles and are in good agreement with the data of other authors [10, 18, 19].

Secondly, the solution of this model problem by classical methods of linear thermal conductivity is necessary to address a much more challenging task of modeling of heat transfer processes in the radiation treatment of coatings and can serve as a criterion for the suitability of one or the other approximate method, the correctness of which can be verified using the model.

\section{Acknowledgments}

This research was funded by a grant (No. 389 (188-418-11) ot 10.02.2012) from the National Agency of Technology Development of Kazakhstan for the theme "Development of technology for surface modification by irradiation to produce nanostructured multifunctional protective coatings with high performance properties."

The authors gratefully acknowledge Prof. A.D. Pogrebnjak (Sumy State University, Sumy, Ukraine) for carrying out the irradiation of coatings by the specified modes and a series of tests of the modified samples, as well as for the discussion of the received results.

\section{References}

[1] D.L. Alontseva, Modification by Irradiation of Ni and Co Based Protective Coatings and Alloys, Monograph, LAP LAMBERT Academic Publishing, Saarbrücken 2012 (in Russian).

[2] D.L. Alontseva, A.L. Krasavin, A.D. Pogrebnjak, S.Z. Rakhmetullina, A.V. Russakova, J. Adv. Mater. 1, 5 (2013) (in Russian). 
[3] D. Alontseva, S. Missevra, A. Russakova, J. Mater. Sci. Eng. 3, 41 (2013).

[4] D. Alontseva, A. Russakova, Adv. Mater. Res. 702, 94 (2013).

[5] D. Alontseva, A. Krasavin, A. Pogrebnjak, A. Russakova, Acta Phys. Pol. A 123, 867 (2013).

[6] H. Carslaw, D. Jaeger, Conduction of Heat in Solids, Clarendon Press, Â Oxford 1959.

[7] A.S. Galitsin, A.N. Zhukovsky, Integral Transforms and Special Functions in Heat Transfer Problems, Naukova Dumka, Kiev 197.

[8] E.A. Airyan, S.I. Bastrukov, M.S. Kaschiev, S.A. Korenev, D.V. Podgainyi, I.V. Puzynin, A.V. Fedorov, A.M. Chervyakov, Russ. Phys. J. 41, 13 (1998).

[9] N.I. Pischasov, A.V. Nikolaev, Commun. Omsk Univ. 2, 39 (1996).

[10] V.E. Ovcharenko, Yu.F. Ivanov, Bull. Tomsk Polytechn. Univ. 3, 88 (2008) (in Russian).

[11] O.P. Solonenko, A.A. Golovin, V.E. Ovcharenko, Bull. Tomsk Polytechn. Univ. 2, 90 (2009) (in Russian).
[12] R. Stanowski, O. Voznyy, J.J. Dubowski, J. Las er Micro/Nanoeng. 1, 17 (2006).

[13] A.G. Maslovskaya, A.V. Sivunov, Comput. Res. Model. 4, 767 (2012) (in Russian).

[14] A. Gnatowski, T. Stachowiak, J. Achiev. Mater. Manufact. Eng. 44, 57 (2011).

[15] R. Blahut, Fast Algorithms for Digital Signal Processing, Mir, Moscow 1989.

[16] M.A. Lavrentiev, B.V. Shabat, Methods of the Theory of Functions of a Complex Variable, Nauka, Moscow 1987.

[17] A.N. Tikhonov, A.A. Samarskiy, The Equations of Mathematical Physics, Nauka, Moscow 1977.

[18] Yu.F. Ivanov, E.V. Nadeikin, Russ. Phys. J. 8, 402 (2009).

[19] F.V. Nolfi, Phase Transformation during Irradiation, Metallurgiya, Chelyabinsk 1989. 